\title{
The feasibility of ground-roll noise suppression from 3D seismic data using high-resolution linear Radon transform
}

\author{
Yue Hu, Feng Cheng, Binbin Mi, Zongbo Xu \\ Institute of Geophysics and Geomatics \\ China University of Geosciences \\ Wuhan, China \\ huyue0716@163.com
}

\begin{abstract}
Ground-roll is a main type of strong noises in petroleum seismic exploration. In oil exploration, ground rolls are treated as some kind of noise contaminating the effective waves. The typical characteristics that enable us to separate surface waves from body waves lie in their difference in velocity and frequency. Significant different features shown in the frequency-velocity (f-v) domain make it possible to separate ground roll noise and effective waves effectively. Ground roll noise suppression in the $f-v$ domain using high resolution linear Radon transform (LRT) is well-established for two-dimension (2D) seismic data (in-line source and receiver spread). Similarly, ground-roll suppression is possible using $2 \mathrm{D}$ wavefield transform (e.g., high-resolution linear Radon transform) from 3D seismic data acquired with any acquisition scheme. Theoretical test demonstrates the feasibility of ground-roll noise suppression for three-dimension (3D) seismic data acquired with random acquisition scheme using the proposed method.
\end{abstract}

Keywords-Ground-roll suppression, frequency-velocity domain, $2 \mathrm{D}$ wavefield waveform, high-resolution Radon transform, $3 D$ seismic data

\section{INTRODUCTION}

Ground roll (the vertical component of Rayleigh wave) is a secondary wave that travels along the free surface, such as the earth-air interface or the earth-water interface. It has the significant characteristics of relatively low velocity, low frequency, high amplitude and strong energy. During data acquisition, ground-roll noise can be suppressed by using proper receiver array design and filtering method. Unfortunately, the effective signals of the overlapped frequency bands could be damaged when ground-roll noise is suppressed. It would result in an unacceptable deterioration of reflections.

Conventional methods for ground-roll noise suppression can be divided into two groups. The first one can be summarized to filter method which is based on suppression of undesired parts of recorded data in the spectral domain, including high-pass and band-pass filtering, f-k filtering and the adaptive ground-roll attenuation method ${ }^{[1-4]}$. The other one is wave field separation method based on ground-roll noise extraction and arithmetical subtraction of it from the raw shot gather, including Wiener-Levinson algorithm, KarhunenLoève (K-L) transform, wavelet transform and Radon

\author{
Jianghai Xia \\ Hubei Subsurface Multi-scale Imaging Key Laboratory \\ China University of Geosciences \\ Wuhan, China \\ jxia@cug.edu.cn
}

transform ${ }^{[5-8]}$. They were developed either on the frequency or the apparent velocity characteristics of ground-roll noise. With only one single characteristic of signals is focused, however, the effective waves would be unavoidably damaged in some degree.

The high-resolution linear Radon transform (LRT) has been advocated for wave field separation for many years ${ }^{[9-13]}$. Luo et al. utilized high-resolution LRT to image the Rayleighwave dispersive energy and separate the multi-mode dispersive Rayleigh-wave energy. It has been demonstrated that high-resolution LRT method is able to achieve much higher resolution of dispersion energy in the $\mathrm{f}-\mathrm{v}$ domain than other algorithms. Ground roll noise suppression in the $f-v$ domain using LRT is well-established for two-dimension (2D) seismic data (in-line source and receiver spread) ${ }^{[14]}$.

Three-dimensional seismic surveys have been applied in oil exploration to handle some complex geological problems. In this study, hence, we focus on extending the techniques to suppress the ground-roll noise from $2 \mathrm{D}$ seismic data to $3 \mathrm{D}$ seismic data sets. 3D seismic surveys can be acquired using different acquisition geometries. It is feasibility to spread 3D records in a linear alignment according to their offsets, and apply high-resolution LRT to extract and suppress ground-roll noise, as it is done for 2D seismic data.

In this paper, we outline the proposed high-resolution LRT and the process of ground-roll noise extraction and suppression for 3D seismic data. Theoretical test in 3D isotropic media has illustrated the feasibility of the proposed method. Since the offsets of receivers are uneven, the wavefield transforms used must be suitable for nonevenly spaced traces. Our results demonstrated that high-resolution LRT is not sensitive to nonevenly spaced records.

\section{HIGH-RESOLUTION LRT}

The forward LRT from the time-offset (t-x) domain to the intercept-slowness $(\tau-\mathrm{p})$ domain can be achieved by summing over amplitudes along linear trajectories

$$
m(p, \tau)=\sum_{x_{\min }}^{x_{\max }} d(x, t=\tau+p x)
$$


where $d(x, t)$ denotes the shot gather (recorded data), $\mathrm{x}$ is the offset between source and receivers, and $t$ is the time, $m(p, \tau)$ is the Radon panel with $p$ denotes the discrete slowness and $\tau$ denotes the zero offset intercept time ${ }^{[3]}$.

Similarly, the conjugate transform from the interceptslowness $(\tau-\mathrm{p})$ domain to the time-offset $(\mathrm{t}-\mathrm{x})$ domain involves summing along the slowness

$$
d(x, t)=\sum_{p_{\min }}^{p_{\max }} m(p, \tau=t-p x)
$$

After taking a temporal Fourier transformation, the LRT can be calculated for each temporal frequency component $\mathrm{f}$. In matrix notation, they can be written as follows,

$$
\mathbf{d}=\mathbf{L} \mathbf{m}
$$

Similarly, the adjoint transformation can be written as

$$
\mathbf{m}_{\text {adj }}=\mathbf{L}^{\mathrm{T}} \mathbf{d}
$$

In Eq.(3), is the forward LRT operator. Based on the matrix theory, asLis not a unitary operator andLTdoes not define the inverse operator, madj denotes a low resolution Radon panel using the transpose or adjoint operator LT.In order to obtain better resolution in the Radon panel, we first define an objective function. The inverse LRT operator can be found by minimizing the following objective function ${ }^{[15-16]}$.

$$
J=\left\|\mathbf{W}_{\mathbf{d}}\left(\mathbf{d}-\mathbf{L} \mathbf{W}_{\mathbf{m}}^{-1} \mathbf{W}_{\mathbf{m}} \mathbf{m}\right)\right\|^{2}+\lambda\left\|\mathbf{W}_{\mathbf{m}} \mathbf{m}\right\|^{2}
$$

In Eq. (5), Wdis a matrix of diagonal weights, Wmis a matrix of model space weights that plays an extremely importantrole in the resolution and smoothness and $\lambda$ maintains balance between data misfit and model constraints. The minimization of the objective function $\mathrm{J}$ can be achieved by solving the following equation using a conjugate gradient(CG) algorithm. More details can be found in many articles ${ }^{[17-18]}$.

$$
\left(\mathbf{W}_{\mathbf{m}}^{-\mathrm{T}} \mathbf{L}^{\mathrm{T}} \mathbf{W}_{\mathbf{d}}^{\mathrm{T}} \mathbf{W}_{\mathbf{d}} \mathbf{L} \mathbf{W}_{\mathbf{m}}^{-1}+\lambda \mathbf{I}\right) \mathbf{W}_{\mathbf{m}} \mathbf{m}=\mathbf{W}_{\mathbf{m}}^{-\mathrm{T}} \mathbf{L}^{\mathrm{T}} \mathbf{W}_{\mathbf{d}}^{\mathrm{T}} \mathbf{W}_{\mathbf{d}} \mathbf{d}(6)
$$

where I denotes the identity matrix, the weighting and the preconditioning matrices Wdand Wmat the ith iteration of the CG algorithm are determined by using diagonal matrices: $\operatorname{diag}\left(\mathbf{W}_{\mathbf{d}}\right)_{\mathrm{i}}=\left|\mathrm{r}_{\mathrm{i}}\right|^{-1 / 2}$ and $\operatorname{diag}\left(\mathbf{W}_{\mathrm{m}}\right)_{\mathrm{i}}=\left|\mathrm{m}_{\mathrm{i}}\right|^{1 / 2}$, where $\mathrm{m}_{i}$ is the Radon model at ith iteration of the CG algorithm, $r_{i}$ is the standard deviation of residual, $\mathbf{r}=\mathbf{L} \mathbf{W}_{\mathrm{m}}^{-1} \mathbf{W}_{\mathrm{m}} \mathbf{m}-\mathbf{d}$. The choice of the range and the interval of $\mathrm{p}$ should avoid aliasing for reconstructing the original signal. Therefore, the highresolution Radon transform can be achieved by using a weighted preconditioned CG algorithm to solve the Eq. (6). Besides, a linear interpolation operation is applied to transform the Radon panel from the frequency-slowness (f-p) domain to the $\mathrm{f}-\mathrm{v}$ domain.

\section{NUMERICAL TEST}

To test the feasibility of the proposed method, a four-layer model (Table. 1) in 3D isotropic media is applied. The source is a $30-\mathrm{Hz}$ Ricker wavelet with $30-\mathrm{ms}$ delay, located at the origin of the polar coordinate. 120 vertical-component receivers are randomly laid in polar coordinates between $45 \mathrm{~m}$ and $1010 \mathrm{~m}$ (Fig. 1a). The total recording length is $2500 \mathrm{~ms}$, with a sampling rate of $1 \mathrm{~ms}$. We spread all receivers in a linear alignment according to their offsets ${ }^{[19]}$. The receiver spatial domain, which represents the random acquisition spread, is spread onto a conventional receiver offset domain (Fig. $1 \mathrm{~b}$ and 1c). In the receiver offset domain, the seismic data is associated with receivers inline with the source. The ground-roll can, hence, be suppressed by applying 2D wavefield transforms to the inline data, as it is done for the traditional inline acquisition schemes. 2D common source gather (Fig. 2a) is obtained, which can be processed using 2D wavefield transforms (e.g., Radon domain), instead of the 3D transforms (e.g., f-kx-ky domain) that are commonly used in microtremor analysis. It allows the problem dimensionality to be reduced from $t-x-y$ domain to $t-x$ domain, thus save considerable computational resources and time. Reflections are weak and contaminated by the existence of strong groundroll noise.

TABLE 1 PARAMETERS OF FOUR-LAYER MODELS

\begin{tabular}{ccccc}
\hline $\begin{array}{c}\text { layer } \\
\text { numbe }\end{array}$ & $\begin{array}{c}\boldsymbol{V p} \\
\boldsymbol{r}\end{array}$ & $\begin{array}{c}\boldsymbol{V} \boldsymbol{c} \\
(\mathrm{m} / \mathrm{s})\end{array}$ & $\begin{array}{c}\text { Densit } \\
(\mathrm{m} / \mathrm{s})\end{array}$ & $\begin{array}{c}\mathrm{kg} / \mathrm{m}^{3} \\
\text { Thickne } \\
\text { ss }(\mathrm{m})\end{array}$ \\
1 & 1000 & 570 & 2100 & 200 \\
2 & 2200 & 1320 & 2250 & 500 \\
3 & 3300 & 2045 & 2400 & 700 \\
4 & 4370 & 2800 & 2560 & Infinite \\
\hline
\end{tabular}



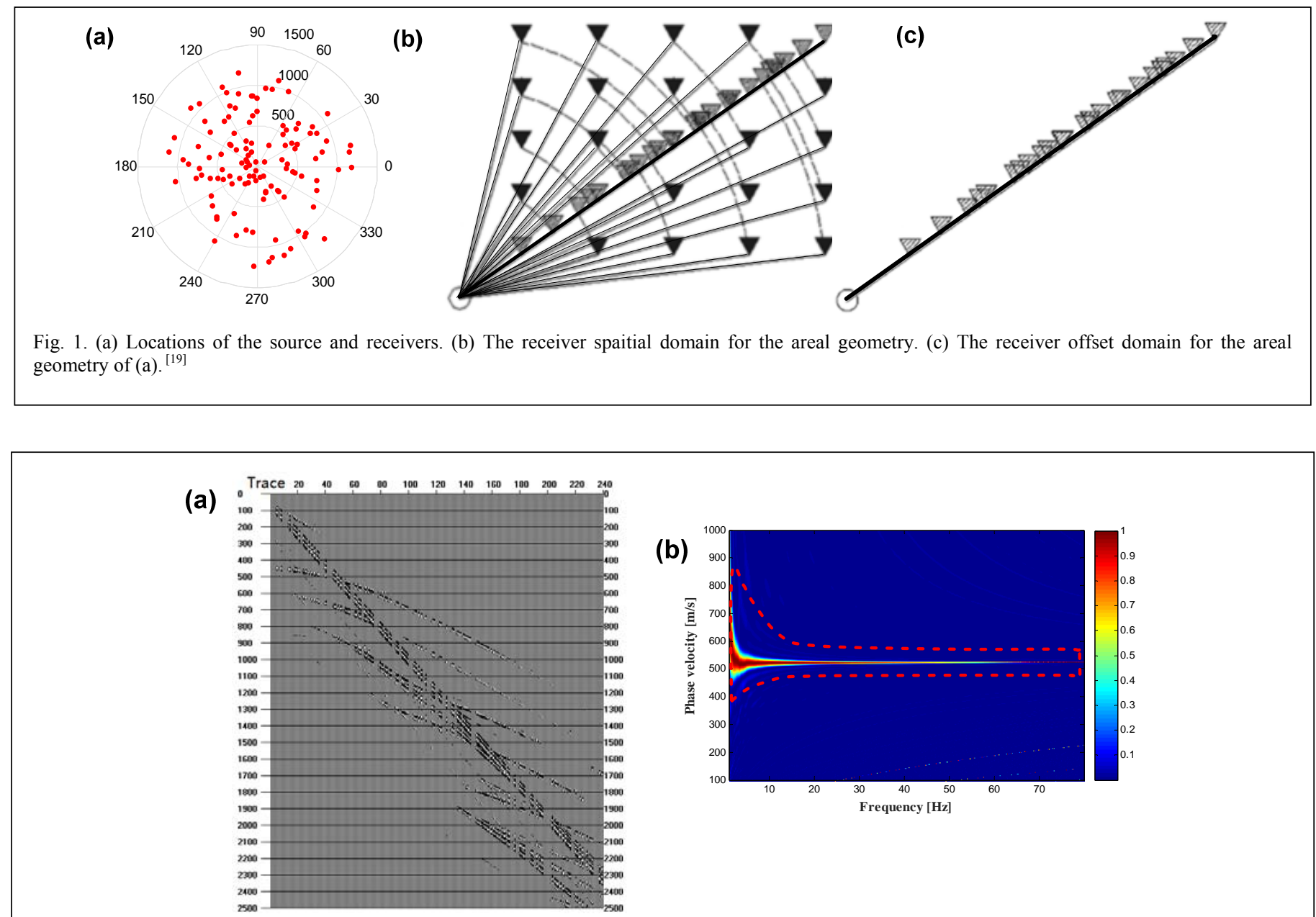

(c)

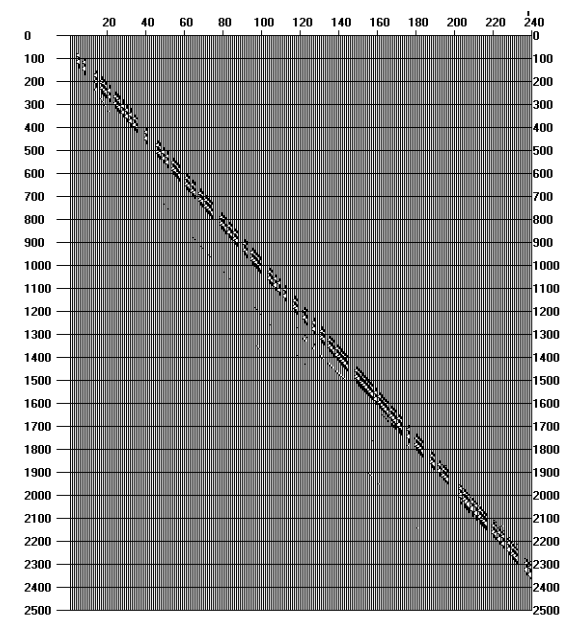

(d)

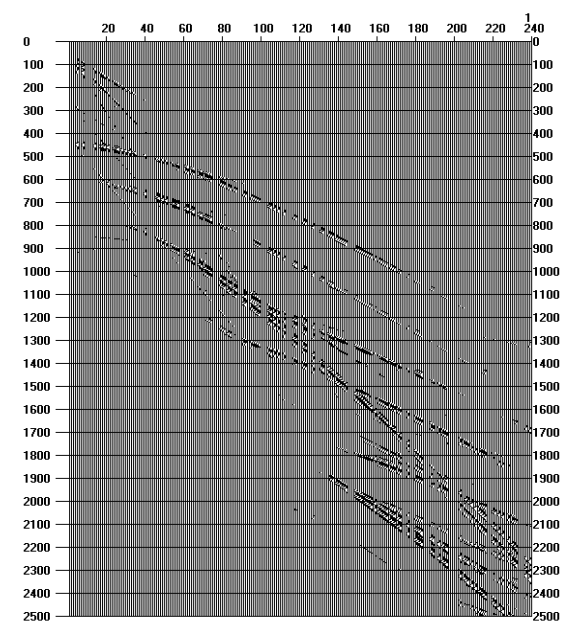

Fig. 2. (a) The synthetic shot gather based on the model in Table 1. (b) The dispersion energy image generated by the high resolution LRT (The red dashed line is the range of the ground-roll that we selected). (c) The extracted ground-roll noise using high-resolution LRT. (d) Effective waves by arithmetically subtracting the extracted ground-roll noise from the raw shot gather (Fig. 2a).

As described previously, the shot gather is first transformed from the $t-x$ domain to the $f-v$ domain by using high-resolution LRT. The obtained dispersion energy image (Fig. 2b) provides sufficient resolution, which is essential to identify ground-roll noise from effective waves completely in the $f-v$ domain. The thickness of the surface layer which is much higher than the Rayleigh waves wavelengths can be approximately to the homogenous half-space. The dispersive 
feature is not obvious. The energy of the ground-roll noise is so strong that the weak reflections are not detectable. According to the distinct different features of ground-roll and reflections, however, we can still identify and select the energy of ground-roll noise accurately in the $f$-v domain, as the red dashed line indicates. The energy outside the selected zone is blanked with a Hanning window tapering along the boundary. Next, we transform the selected ground-roll noise energy back to the $t-x$ domain using the inverse LRT to obtain the extracted ground-roll noise (Fig. 2c). Finally, the extracted ground-roll noise (Fig. 2d) is subtracted arithmetically from the raw seismic data in the $t-x$ domain (Fig. 2a) to obtain the objective waves (Fig. 2d). Fig. $2 d$ indicates the ground-roll noise is efficiently suppressed and the reflection events are more coherent in $500 \mathrm{~ms}, 800 \mathrm{~ms}, 1200 \mathrm{~ms}$, and $2000 \mathrm{~ms}$ without the interference of ground-roll. The theoretical test reveals that the ground-roll noise is effectively suppressed without damage of effective waves using the proposed method.

\section{CONCLUSIONS}

In this preliminary study, we analyze the feasibility of ground-roll noise suppression for 3D seismic data using highresolution linear Radon transform. It is valid to spread 3D records in a linear alignment according to their offsets, and apply high-resolution LRT to extract and suppress ground-roll noise, as it is done for 2D seismic data.

Since the offsets of receivers are uneven, the wavefield transforms used must be suitable for nonevenly spaced traces. We prove that high-resolution LRT is not sensitive to nonevenly spaced records. Further studies will be required verify the feasibility of this method in laterally varying media, as well as real word 3D seismic data.

\section{ACKNOWLEDGMENT}

This study is supported by the National Natural Science Foundation of China (NSFC, Grant No. 41274142) and the National Nonprofit Institute Research Grant of Institute for Geophysical and Geochemical Exploration, Chinese Academy of Geological Sciences (Grant No. WHS201306).

\section{REFERENCES}

[1] Embree, P., Burg, J.P., Backus, M.M., 1963. Wide band velocity filtering-the pie-slice process. Geophysics 28, 948- 974.

[2] Treitel, S., Shanks, J.L., Fraster, C.W., 1967. Some aspects of fan filtering. Geophysics 32, $789-800$.

[3] Yilmaz, Ö, 2001, Seismic data analysis: Society of Exploration Geophysicists, Tulsa, Oklahoma.

[4] Hosseini, S. A., Javaherian, A., Hassani, H., Torabi, S., and Sadri, M., 2015, Adaptive attenuation of aliased ground roll using the shearlet transform: Journal of Applied Geophysics, 112, 190-205.

[5] Karsl1, H., and Bayrak, Y., 2004. Using the Wiener-Levinson algorithm to suppress ground-roll: Journal of Applied Geophysics 55(3), 187-197.

[6] Liu X., 1999, Ground roll suppression using the Karhunen-Loève transform: Geophysics, 64(2):564-566.

[7] Benoliel, S.D., Schneider, W.A., and Shurtleff, R.N., 1987, Frequency wavenumber approach of the t-p transform: some applications in seismic data processing: Geophysical prospecting, 35(5), 517-538.

[8] Tokeshi J. C., and Karkee M. B., and Sugimura. Y., 2006, Reliability of Rayleigh wave dispersion curve obtained from $\mathrm{f}-\mathrm{k}$ spectral analysis of micro-tremor array measurement: Soil dynamics and earthquake engineering, 26(2), 163-174.

[9] Foster, D. J., and Mosher, C. C., 1992, Suppression of multiple reflections using the Radon transform: Geophysics, 57(3), 386-395.

[10] Luo, Y., Xia, J., Miller, R.D., Xu, Y., Liu, J., and Liu, Q., 2008, Rayleigh-wave dispersive energy imaging using a high-resolution linear Radon transform: Pure and Applied Geophysics, 165(5), 903-922.

[11] Luo, Y., Xia, J., Miller, R.D., Xu, Y., Liu, J., and Liu, Q., 2009, Rayleigh-wave mode separation by high-resolution linear Radon transform: Geophysical Journal International, 179(1), 254-264.

[12] Nowak, E., and Imhof, M., 2006, Amplitude preservation of radonbased multiple-removal filters: Geophysics, 71(5), V123-V126.

[13] Thorson, J. R., 1985, Velocity-stack and slant-stack stochastic inversion: Geophysics, 50(12), 2727.

[14] Hu, Y., Wang, L., Cheng, F., Luo, Y., Shen, C., \& Mi, B., 2016, Ground-roll noise extraction and suppression using high-resolution linear Radon transform: Journal of Applied Geophysics, 128, 8-17.

[15] Trad, D., Ulrych, T. and Sacchi, M., 2002, Accurate interpolation with high-resolution time-variant Radon transforms: Geophysics, 67(2), 644656.

[16] Trad, D., Ulrych, T. and Sacchi, M., 2003, Latest views of the sparse Radon transform: Geophysics, 68(1), 386-399.

[17] Sacchi, M., \& Ulrych, T., 1995, High-resolution velocity gathers and offset space reconstruction: Geophysics, 60(4), 1169-1177.

[18] Ji, J., 2006, CGG method for robust inversion and its application to velocity stack inversion: Geophysics, 71(4), R59-R67.

[19] Boiero, D., Bergamo, P., Bruno Rege, R., \& Socco, L. V., 2011, Estimating surface-wave dispersion curves from $3 \mathrm{~d}$ seismic acquisition schemes: part 1 - 1d models: Geophysics, 76(6), G85-G93. 